\title{
Intradural extramedullary spinal masses treated at the Wits teaching hospitals between $2014-2017$
}

\author{
JR Ouma \\ Department of Neurosurgery, University of the Witwatersrand, Johannesburg and Chris Hani Baragwanath Hospital, Soweto, \\ South Africa
}

Corresponding author: John Ouma (glioma42@gmail.com)

\begin{abstract}
Background: Intradural extramedullary (IDEM) spinal masses are common lesions with varying histological diagnoses often associated with significant neurological deficits. This study aimed to describe the epidemiology, management and perioperative outcome of IDEM tumours seen at the teaching hospitals of the University of the Witwatersrand, Johannesburg, between 2014 and 2017.

Results: 92 patients were included in the study. The ages ranged from 21 to 87 years, sex ratio was M:F 1:1.4, and duration of symptoms prior to diagnosis ranged between 3 days to 18 months. Local and radicular type pain as well as motor weakness were the commonest symptoms. $67 \%$ had severe neurological deficit McCormick Grade III and IV. Schwannoma (26) Neurofibromas (21) and Meningiomas (16) and were the most frequent tumour types. Meningiomas predominantly occurred at the cranio-cervical and thoracic levels. Nerve sheath tumours were mostly found at the cervical and lumbar levels while filum terminale ependymomas occurred at the thoracolumbar area. Laminectomy was the commonest surgical approach employed, and the extent of resection varied, with total excision in half the cases. Neurological function was regained in 3 patients, deteriorated in two and was unchanged in the remainder.

Conclusions: IDEM tumours are an important subset of spinal cord compressive lesions Presentation with severe neurological deficit is common and though resection is feasible neurological deficit remains in the vast majority. Earlier detection should improve the results of surgery.
\end{abstract}

S Afr J Surg 2019;57(3)

http://dx.doi.org/10.17159/2078-5151/2019/v57n3a2919

\section{Introduction}

Extradural spinal tumours due to vertebral metastases or HIV associated lymphoma in the old and the young respectively are the commonest causes of spinal cord compression. Intradural extramedullary (IDEM) spinal masses are the next in frequency with intramedullary tumours being relatively rare. IDEM occur across the age and race spectrum of our population, with marginal gender predilection in certain tumour types. They are predominantly neoplastic processes, although a small number of non-neoplastic lesions do occur.

\section{Materials and methods}

This paper aims to present a description of the epidemiology and management of IDEM tumours seen at the teaching hospitals of the University of the Witwatersrand, Johannesburg, between 2014-2017. During this period, 92 patients with IDEM tumours were managed surgically. Twenty-one patients were diagnosed radiologically but did not undergo surgery and hence no histological analysis was made. The McCormick classification for IDEM tumours ${ }^{1}$ was used to assess the degree of neurological impairment: Grade 1 neurologically normal, mild focal deficits, normal gait; Grade 2 - sensorimotor deficits affecting function, severe pain, gait difficulties, can still walk; Grade 3 - moderate neurological deficit, requires cane for ambulation, minor involvement of arms possible, patient is partially independent; Grade 4 - as Grade 3 with arms affected, usually not independent. 71 surgically resected specimen were sent for histology. Only perioperative outcomes were recorded as their further care post discharge was conducted at the referring primary hospitals or rehabilitation centres.

\section{Results}

\section{Main symptoms at presentation}

The patients' age ranged between $21-87$ years. The male: female ratio was $1: 1.4$. Most of the patients presented with 
Table 1: McCormick Grade at presentation amongst our patients

\begin{tabular}{lc}
\hline Main clinical symptom & Frequency (\%) \\
\hline Local spinal pain & 27 \\
Radicular pain & 19 \\
Motor weakness & 18 \\
Sensory deficit & 17 \\
Sphincter dysfunction & 15 \\
Erectile dysfunction & 4
\end{tabular}

multiple complaints, the commonest being local pain along the area of the spine where the tumour was, as well as radicular type pain. Motor weakness was a common problem in almost half of cases, while sensory disturbances, sphincter control problems and erectile dysfunction in male patients were much less frequently disclosed.

The majority of patients that we saw came from socioeconomically disadvantaged backgrounds with a symptom duration of 3 days to 18 months (mean 8 months) at the time of presentation. This long history likely accounted for the unfavourable clinical state at presentation in the majority of our patients. Table 1 details the McCormick Grade at presentation.

The vast majority of lesions had a laminectomy approach. Even in instances where the tumour had a ventral or ventrolateral origin relative to the spinal cord, we found in many that lateral displacement of the spinal cord allowed a posterior approach without any mobilization of the cord. Other approaches employed included the posterior fossa craniectomy route for meningiomas of the cranio-cervical area, anterior cervical corpectomy and fusion for ventrally located cervical tumours, and thoracotomy and costotransversectomy for ventral or ventrolateral thoracic tumours respectively. Total excision was possible in $50 \%$ of the cases, with near total ( $>90 \%$ ) excision in a further $38 \%$. Limited decompression was performed in $8.5 \%$ of cases and in $4 \%$, only a biopsy was taken. The main factor determining extent of resection was safety of the spinal cord. Where further surgery would impose an increased risk to the cord and its vasculature due to manipulation, then the procedure was terminated at that point. Additional considerations were stability of the vertebral column. Wound dehiscence and cerebrospinal fluid (CSF) leaks occurred in 4 and 3 cases respectively. Two patients experienced neurological deterioration after their surgeries, dropping one McCormick Grade in each instance, one had wrong level surgery and a further one developed spinal instability necessitating a further stabilising procedure with instrumented fusion.

The location and frequency of the IDEM tumours is depicted graphically in Figure 1. Meningiomas occurred primarily in the thoracic spine. However, a number of foramen magnum meningiomas did extend significantly down the spine to warrant inclusion in this study as IDEM tumours of the cervical spine. Meningiomas also did occur in the lumbar spine. Of the spinal nerve sheath tumours, these were mainly seen in the cervical and lumbar spines. Filum terminale ependymomas occurred exclusively in the lumbar spine area. A closer look at specific tumour types revealed significant differences in age and gender distribution. The peripheral nerve sheath tumours (schwannomas and neurofibromas) tended to occur in the 4th to 5 th decades of life, with an equal gender balance, while the meningiomas were overwhelmingly seen in females $(80 \%)$, and these were patients mainly in the 6th decade of life. Filum terminale ependymomas occurred mainly amongst young adults, and there was a female preponderance of $2: 1$.

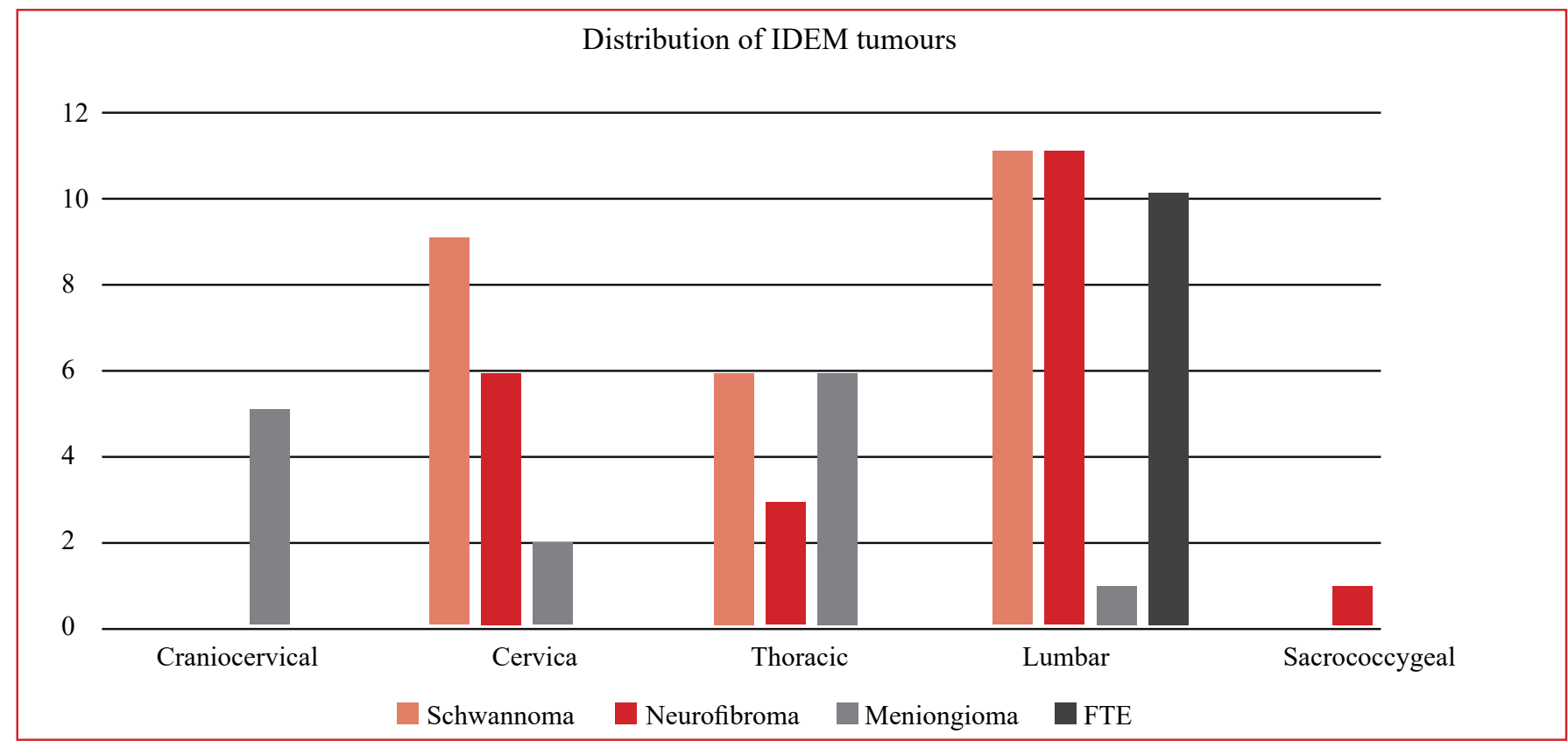

Figure 1: Histogram showing tumour frequency related to location 


\section{Discussion}

Our cohort of patients had features similar to the published literature in as far as age and gender occurrence of IDEM tumours are concerned, as well as main clinical features. ${ }^{2-4}$ The frequency of IDEM tumours as a subset of all spinal tumours varies widely in the literature, as does the relative prevalence of the different types of tumour within this group. ${ }^{2,-6}$ Our patients do, on average, present late. This could be due to the socio-economic challenges prevalent in the demographic of patients that access public hospitals. As a result, neurological deficits are frequently well established at presentation and this tends to affect outcomes. It is imperative that a greater understanding of the early signs and symptoms of spinal cord compression are recognised at the primary hospitals if this situation is to be reversed; several late presenters did in fact report having attended primary care health facilities early in the course of their disease.

If one is to go by our experiences in spinal tuberculosis, where patients on average tend to improve neurologically post-decompression, there is reason to believe that similar outcomes are possible with some of our IDEM patients.

The surgical approach most employed is a laminectomy. This is a familiar procedure and is relatively straightforward, if the tumour is directly accessible without any retraction of the spinal cord. In several instances, we found that the cord was sufficiently mobilised laterally even by tumours that originate ventrally, allowing laminectomy based surgery from a posterior approach. ${ }^{5}$

Where this is not the case, however, then more anterior approaches are called into play; these include the anterior cervical corpectomy and fusion, as well as the costotransversectomy and transthoracic approaches to the thoracic spine. These approaches require a higher skill set and equipment than does a laminectomy.

Overall, as these figures show, the rate of serious complications in this series was low.

CSF leaks are predisposed to by larger tumours, greater degrees of dural resection and the associated difficulty in repairing the resultant dural defects. They tended to occur in females with large thoracic meningiomas in the main. We now tend to use temporary lumbar drains liberally in such cases, in addition to watertight closure of the soft tissues.

Wrong level surgery occurred in one case, caused by wrong identification of lumbar vertebral levels by relying on MRI rather than X-rays for this purpose. Spinal instability requiring a second stabilising procedure occurred in one case in which the extent of bony resection was underestimated intraoperatively.

Neurological status tended to remain unchanged in most patients over the period of a couple of weeks follow-up prior to discharge that obtained in this cohort of patients. The two patients in whom there was a clear drop in neurological status postoperatively had presented with high Grade 3 McCormick neurological states and long duration of complaints. In these cases, it is likely that the reserve of the spinal cord to tolerate any further insult is exhausted and so what little surgical trauma occurs becomes magnified. This explanation finds resonance in the published literature. , $^{2,3}$

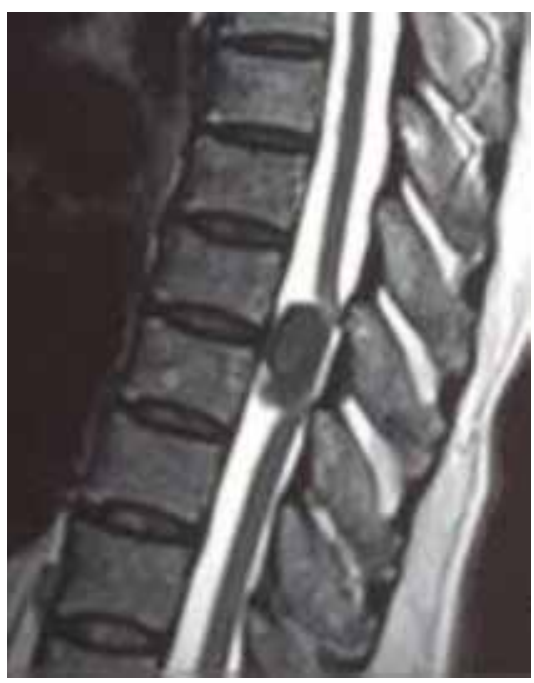

Figure 2: T2 weighted sagittal MRI scan of the thoracic spine showing an IDEM meningioma

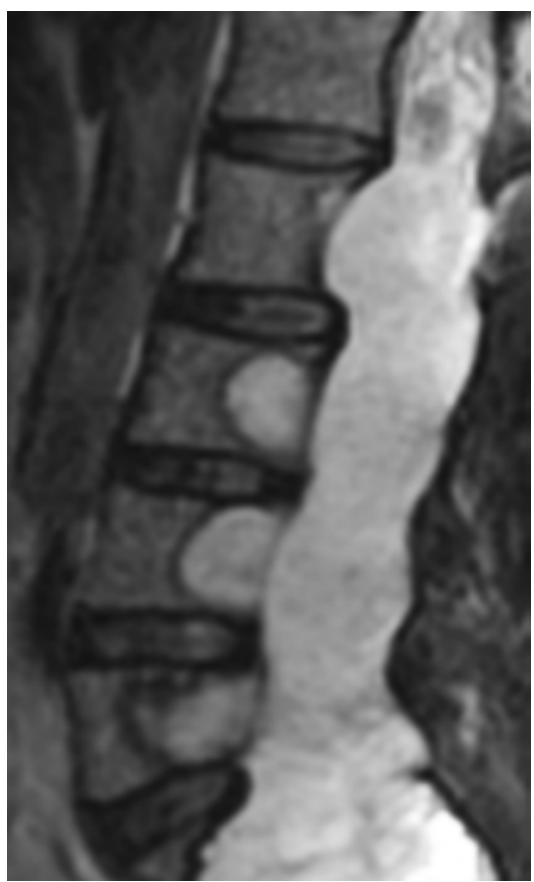

Figure 3: T2 weighted sagittal MRI scan of the lumbar spine showing a myxopapillary ependymoma

\section{Conclusions}

IDEM tumours represent an important subset of spinal pathology whose outcomes depend on neurological status at presentation, proper workup and judicious management. Severe neurological deficits at presentation are common place amongst the low socio-economic communities that tend to use public hospitals in our setting, hence greater efforts are necessary to identify these cases earlier and treat them timeously in this demographic. While several cases are amenable to straightforward surgical approaches, the skill set required for the safe management of the more difficult cases precludes treatment at small volume centres.

\section{REFERENCES}

1. Kumar R. Management and functional outcome of intramedullary tumors in adults. McCormick PC SBISD, McCormick PC, eds. Neurosurgery Clinics in North America: Intradural Spinal Surgery. 1990;1:609-30. [PubMed]

2. Byvaltsev VA, Damdinov BB, Belykh EG, Ivanova KO, 
Sorokovikov VA, Tsunetoshi K, et al. Neurosurgical Outcomes of Intradural Extramedullary Spinal Tumors in 97 cases:Siberian Experience. No shinkei geka Neurological surgery. 2017;45(9):781-7.

3. $\mathrm{Ng} \mathrm{Z,} \mathrm{Ng} \mathrm{S,} \mathrm{Nga} \mathrm{V,} \mathrm{Teo} \mathrm{K,} \mathrm{Lwin} \mathrm{S,} \mathrm{Ning} \mathrm{C,} \mathrm{et} \mathrm{al.} \mathrm{Intradural}$ Spinal Tumors - Review of Postoperative Outcomes Comparing Intramedullary and Extramedullary Tumors from a Single Institution's Experience. World Neurosurg. 2018;109:e229-e32.

4. Honda A, Iizuka Y, Hirato J, Kiyohara H, Iizuka H. Multiple intradural-extramedullary spinal ependymomas including tumors with different histological features. Eur Spine J. 2017;26(Suppl 1):222-4.

5. Chikani MC, Okwunodulu O, Mesi M, Mezue WC, Ohaegbulam SC, Ndubuisi CC. Surgically Treated Primary Spinal Cord Neoplasms in Southeastern Nigeria. J Neurosci Rural Pract. 2018;9(1):137-9.

6. Chakravorty A, Frydenberg E, Shein TT, Ly J, Earls P, Steel T. Multifocal intradural extramedullary anaplastic ependymoma of the spine. J Spine Surg (Hong Kong). 2017;3(4):727-31. 